Wir arbeiteten bei beginnender Weissglühhitze, aber es konnte keine nennenswerthe Verfüchtigung des Magnesiums erzielt werden. Die Versuche, die Dampfdichte des Magnesiums zu bestimmen, müssen daher vorläufig wohl als anssichtslos bezeichnet werden.

Schliesslich sei bemerkt, dass

\title{
An $\mathrm{t}$ i m o $\mathrm{n}$
}

oberhalb $1300^{\circ}$ recht reichlich, aber doch nicht rasch genug verdampft, um eine Gasdichtebestimmung zu ermöglichen. Wir sind daher mit der Construction eines Ofens beschättigt, welcher uns hoffentlich erlauben wird, die Dampfdichte dieses Grundstoffs bei $1600^{\circ} \mathrm{C}$. 20 bestimmen.

Göttingen, Unirersitäts-Laboratorium.

\section{Victor Meyer und A. Warrington: Zur Kenntniss der Acetoxime.}

(Eingegangen am 18. Februar; mitgetheilt in der Sitzung ron Hrn. F. T ie mann.)

In einer im vorigen Jahre veröffentlichten vorläufigen Mittheilung baben wir den Plan der folgenden Arbeit und die ersten orientirenden Versuche kurz mitgetheilt. Damals wurde etwa Folgendes gesagt': Fast alle Aldoxime geben mit Acetylchlorid Nitrile nach der Gleichung:

$$
\text { R. } \mathrm{CH}: \mathrm{N} \cdot \mathrm{OH}=\mathrm{H}_{2} \mathrm{O}+\mathrm{R} \cdot \mathrm{C}: \mathrm{N} \text {, }
$$

fast alle Acetoxime aber geben mit dem gleichen Reagens Acetyläther der Formel: $\mathrm{R}, \mathrm{C}\left(\mathrm{N}, \mathrm{O}, \mathrm{C}_{2} \mathrm{H}_{3} \mathrm{O}\right), \mathrm{R}_{1}$. Eine Ausnahme bildet bei den Aldoximen dasjenige des Terephtalaldehyds, welches einen Diacetyläther giebt; bei den Acetoximen das Campheroxim, welches in Nitril der Campholensäure verwandelt wird. Die letztere Thatsache ist nur dadurcb zu erklären, dass der Campher als eine Art von Additionsproduct der aromatischen Reibe Wasserstoffatome in besonders exponirter Stellung entbält, welche in ibrer Lage mit den Wasserstoffatomen der Aldehyde und der Aldoxime Aehnlichkeit haben. Danach drängte sich die Frage auf, ob das Campheroxim und das Terephtalaldoxim allein stehende Ausnabmen seien, oder ob sie Analogien haben. Die Untersuchuug des Isophtalaldoxims, welche Hr. Münchmeyer auf Veranlassung des Einen von uns vor Kurzem ausgeführt, bat gezeigt, dass das Analogon des Terephtalaldoxims diesem durchaus nicht 
gleicht. Es gab Metadicyanbenzol; eine Verschiedenbeit im Verhalten zweier so ähnlicher Körper, die vorläufig ganz unerklärbar ist.

Um ein Analogon des Campheroxims von genauer bekannter Constitution, als es bei dem Derivate der immer noch problematischen Camphergruppe der Fall ist, untersuchen zu können, wandten wir uns. zu Acetoximen mit tertiären Wasserstoffatomen. Richard Meyer hat die besonders angreifbare Natur solcher Wasserstoffatome erwiesen; ihre Stellung scheint uns mit denjenigen der an das Benzol addirten Wasserstoffatome wohl vergleichbar. Wir theilten schon damals mit, dass die beiden Acetoxime:

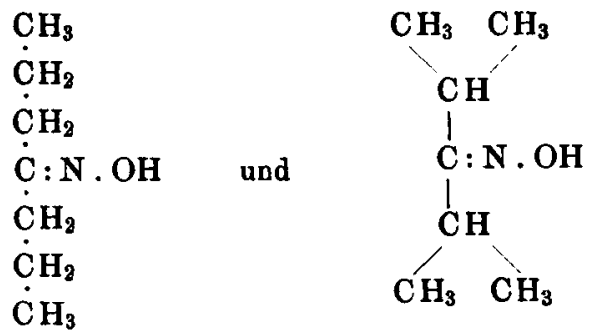

also das Dipropyl- und das Diisopropylacetoxim, sich gegen Chloracetyl ganz verschieden verbalten. Die nähere Untersuchung dieser Verbältnisse bildet den Gegenstand unserer heutigen Mittheilungen.

\section{Dipropylacetoxim.}

Dipropylketon, leicht zu erhalten durch Destillation von buttersaurem Kalk, wurde in bekannter Weise durch ein- oder mehrtägiges Kochen mit alkalischer Hydroxylaminlösung in sein Oxim verwandelt. Das letztere bildet eine farblose Flüssigkeit, welche zwischen 190 und $195^{\circ} \mathrm{C}$. siedet, einen starken und charakteristischen Geruch besitzt und in einer Kältemischung von Eis und Kochsalz nicht erstarrt. Die Analyse ergab:

$0.2069 \mathrm{~g}$ gaben $19.7 \mathrm{~cm}$ feuchten Stickstoff bei $15.5^{\circ} \mathrm{C}$. und $746.3 \mathrm{~mm}$ Druck.

$$
\begin{array}{ccc} 
& \text { Berechnet } & \text { Gefunden } \\
\text { N } & 10.85 & 10.92 \mathrm{pCt} .
\end{array}
$$

Das Acetoxim wurde in einen grossen Ueberschuss von Acetylchlorid eingetragen - die Verdünnung durch das überschüssige Chlorid verhindert, dass die Reaction zu stürmisch wird - dann das Gemisch erwärmt und schliesslich in kaltes Wasser oder Eis gegossen. Es scheidet sich ein ätherisch riechendes Oel ab, das durch Aether extrabirt und im Vacuum über Schwefelsäure getrocknet wird. Dasselbe ist der erwartete Acetyläther $\mathrm{C}_{3} \mathrm{H}_{7} . \mathrm{C}\left(\mathrm{N} . \mathrm{O} \cdot \mathrm{C}_{2} \mathrm{H}_{3} \mathrm{O}\right) . \mathrm{C}_{3} \mathrm{H}_{7}$.

$0.2720 \mathrm{~g}$ gaben $18.5 \mathrm{ccm}$ feuchten Stickstoff bei $16^{\circ} \mathrm{C}$. und $752 \mathrm{~mm}$ Druck.

$$
\begin{array}{ccc} 
& \text { Berechnet } & \text { Gefunden } \\
\text { N } & 8.00 & 7.91 \mathrm{pCt} .
\end{array}
$$


Eine Umwandlung dieses Aethers, anhnlich der seines im folgenden Abschnitte zu beschreibenden Isomeren, haben wir nicht beobachtet, obwohl wir den Körper in ganz analoger Weise behandelt haben.

Di isopropylacetoxim.

Das erforderliche Diisopropylketon wurde durch Destillation ron isobuttersaurem Kalk gewonnen. Mit Hydroxylamin in der oben angegebenen Weise bebandelt, geht es leicht in sein Oxim über. Dies bildet ein farbloses, sehr stark und charakteristisch, nicht unangenehm riechendes Oel, welches zwischen 181 und $185^{\circ} \mathrm{C}$. siedet und in einer Kältemischung zu Krystallen erstarrt. Diese letzteren schmelzen zwischen +6 und $+8^{\circ} \mathrm{C}$.

Die Analyse derselben ergab:

$0.1555 \mathrm{~g}$ gaben $14.4 \mathrm{ccm}$ feuchten Stickstoff bei $18.5^{\circ} \mathrm{C}$. und $748 \mathrm{~mm}$ Druck.

$$
\begin{array}{ccc} 
& \text { Berechnet } & \text { Gefunden } \\
\text { N } & 10.85 & 10.48 \mathrm{pCt} .
\end{array}
$$

Wird dieses Oxim in einen grossen Veberschuss ron Chloracetyl sehr vorsichtig eingetragen - die Reaction kann leicht explosionsartig werden - und das Gemisch in Eis gegossen, so erhält man ein Acetylderivat, welches dem im vorigen Abschnitte beschriebenen ăhnlich ist. Destillirt man aber nach dem Eintragen des Oxims das überschüssige Chloracetyl im Wasserbade $a b$ und erwärmt den Rückstand längere Zeit auf dem Wasserbade, so ist der Anfangs gebildete Aether verschwunden. Denn giesst man nunmebr die Masse in Wasser oder Eis, so scheidet sich nichts ab, sondern man erbält eine völlig klare Lösung. Fügt man zu der erwärmten Lösung Natronlauge oder festes Natron, so steigt ein braunes Oel an die Oberfläche, welches einen betäubenden Alkaloïdgeruch besitzt. Die Untersuchung dieses Oeles hat nun merkwürdige und unerwartete Resultate ergeben. Dasselbe besitzt keinen constanten Siedepunkt, erleidet aber bei der Destillation keine erhebliche Zersetzung. Während des Destillirens, ja auch beim längeren Aufbewahren des nicht destillirten Oeles, bemerkt man eine reichliche Abscheidung von Krystallen. Diese lässt sich sehr vermehren, wenn man das Oel auf dem Boden grosser, flacher Glasschalen ausbreitet und längere Zeit stehen lässt. Bald scheint das Ganze erstarrt, aber beim Absaugen der Krystalle erhält man doch eine beträchtliche Menge öliger Mutterlauge. Diese wird wieder ausgebreitet stehen gelassen, worauf sie von Neuem Krystalle abscheidet. So gelang es im Laufe von Wochen, die Menge des Oeles immer mehr und mebr zu verkleinern und das Meiste in Krystalle zu verwandeln, die schliesslich zwischen Filtrirpapier scharf ausgepresst und aus lauwarmem Wasser umkrystallisirt werden.

Die reinen so erhaltenen Krystalle erwiesen sich nun zu unserer Ueberraschung als vollkommen geruchlos. Der intensive Alka- 
loïdgeruch ist offenbar nicht den Krystallen, sondern dem Oel zuzuschreiben, dessen letzte Spuren schliesslich durch das Abpressen und Umkrystallisiren entfernt worden waren. Der neue Körper ist eine Substanz von ausserordentlich einladenden Eigenschaften. Er bildet weisse, farblose Nadeln, welche in Alkohol und Aether sehr leicht löslich sind, in Wasser gerade so leicht, um bequem daraus um krystallisirt werden zu können. Er ist sehr flüchtig, denn, ähnlich dem Naphtalin, sublimirt er beim Aufbewahren schon bei Zimmertemperatur in grossen, perlmutterglänzenden, federförmigen Krystallen. Er schmilzt bei $102^{\circ} \mathrm{C}$. und siedet constant and unzersetzt bei $210^{\circ} \mathrm{C}$.

Seine Analyse ergab folgende Resultate:

I. $0.1587 \mathrm{~g}$ Substanz gaben $15.20 \mathrm{ccm}$ feuchten Stickstoff bei $21^{\circ} \mathrm{C}$. und $742.5 \mathrm{~mm}$ Druck.

II. $0.1334 \mathrm{~g}$ Substanz gaben $12.2 \mathrm{cem}$ feuchten Stickstoff bei $17^{\circ} \mathrm{C}$. und $754.5 \mathrm{~mm}$ Druck.

III. $0.2186 \mathrm{~g}$ Substanz gaben $0.5198 \mathrm{~g}$ Kohlensăure und $0.2301 \mathrm{~g}$ Wasser.

$\begin{array}{lcccc}\text { Ber. für } \mathrm{C}_{7} \mathrm{H}_{15} \mathrm{NO} & \text { I. } & \text { II. } & \text { III. } \\ \mathrm{C} & 65.12 & - & - & 64.94 \mathrm{pCt} . \\ \mathrm{H} & 11.63 & - & - & 11.74 \text {, } \\ \mathrm{N} & 10.85 & 10.65 & 10.53 & -\end{array}$

Diese Zablen führen zu dem unerwarteten Ergebniss, dass der Körper dieselbe Zusammensetzung hat, wie das angewandte Acetoxim, während die Eigenschaften beider Substanzen so verschieden wie möglich sind. Dass auch die Moleculargrössen identisch sind, beweisen die folgenden Dampfdichtebestimmungen:

Die Bestimmungen geschahen nach der Methode von $V$. und $C$. Metger im Thymoldampf.

I. $0.0464 \mathrm{~g}$ Substanz verdrăngten $8.8 \mathrm{~cm}$ Luft bei $24.5^{\circ} \mathrm{C}$. und $741.3 \mathrm{~mm}$ Druck.

II. $0.0508 \mathrm{~g}$ Substanz verdrängten $9.8 \mathrm{ccm}$ Luft bei $24.5^{\circ}$ C. und $741.3 \mathrm{~mm}$ Druck.

III. $0.0396 \mathrm{~g}$ Substanz verdrängten $7.55 \mathrm{ccm}$ Luft bei $24.5^{\circ} \mathrm{C}$. und $741.3 \mathrm{~mm}$ Druck.

Dampfdichte :

Ber. für $\mathrm{C}_{7} \mathrm{H}_{15} \mathrm{NO}$

\subsection{7}

I. Gefunden

III.

$4.70 \quad 4.62 \quad 4.67 \mathrm{pCt}$.

Legt man sich die Frage nach der Constitution des entstandenen Körpers vor, so drängt sich zunächst der Gedanke auf, dass das Oxim, ähnlich dem Campheroxim, Wasser verliere und einen nitrilartigen Körper $\mathrm{CH}_{3}$ ein sogenanntes Meta-Nitrils. erzeugt habe; dies wäre 
Da aber nach den zahlreichen jetzt vorliegenden Erfahrungen nicht mehr bezweifelt werden kann, dass Metanitrile unbestandig sind und sich da, wo sie entstehen können, immer sogleich verändern (man denke an die Ketine, das Isoindol n. s. w.), so konnte das hypothetische Product im vorliegenden Falle sogleich wieder Wasser aufge-

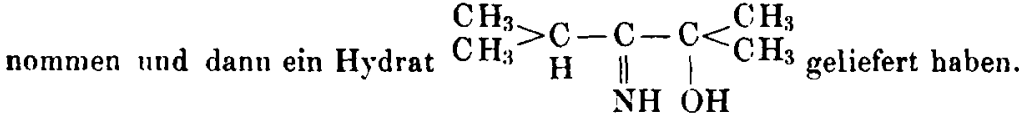

Diese Formel sollte zunächst geprüft werden.

Man versuchte Salze der neuen Base darzustellen. Allein, obwohl in Säure leicht löslich und durch Alkalien aus der Lösung wieder fällbar, giebt sie weder mit Salzsä̈ure noch mit Platinchlorid, weder mit Schwefelsäure noch mit anderen Süuren irgend fassbare Derivate. Es wurde dann versucht, die Imidgruppo mittelst salpetriger Säure nachzuweisen. Jedoch man erhielt kein Nitrosoderivat.

Eine überraschende Aufklärung brachte endlich das Verhalten bei der hydrolytischen Spaltung. Kocht man die Base andauernd nit concentrirtem alkoholischem Kali, oder erhitzt man sie mit Salzsïure in zugeschmolzenem Rohre, so wird sie glatt in

\section{Isobuttersäure und Isopropylamin}

gespalten. Die Isobuttersäure wurde mit Wasserdampf übergetrieben, ins Kalksalz übergeführt und dieses analysirt. Die Analyse ergab:

$0.340 .5 \mathrm{~g}$ des hei $150^{\circ}$ getrockneten Salzes gaben $0.2574 \mathrm{~g}$ Baryumenlfat.

$$
\begin{aligned}
& \text { Ber. für }\left(\mathrm{C}_{4} \mathrm{H}_{7} \mathrm{O}_{2}\right)_{2} \mathrm{Ba} \\
& \text { Gefunden } \\
& \mathrm{Ba} \quad 44.07 \quad 44.43 \mathrm{pCt} \text {. }
\end{aligned}
$$

Das Isopropylamin wurde als Platinsalz isolirt, dessen Analyse zin folgenden Ergebnissen führte:

$0.5988 \mathrm{~g}$ des Platindnppelsalzes gaben nach dem Glühın $0.2260 \mathrm{~g}$ Platin. Berechnet

$$
\text { für } \begin{array}{cc}
\left(\begin{array}{l}
\left.\mathrm{CH}_{3}<\mathrm{CH} . \mathrm{NH}_{2} . \mathrm{HCl}\right)_{2} \mathrm{PtCl}_{4} \\
\mathrm{Pt} \quad 37.74
\end{array}\right. & \text { Gefunder } \\
\mathrm{Ct} & 37.17 \mathrm{pCt} .
\end{array}
$$

Um sicher zu sein, dass die vorliegende Säure nicht normale, sondern Isobuttersäure sei, wurde die bekannte Kalksalzprobe vorgenommen, welche in unzweideutiger Weise für Isobuttersäure entschied.

Welches ist nun die Constitution dieses schönen und durch seine Bildungsweise so merkwürdigen Körpers?

Die eben genannte Spaltung, im Verein mit der überraschenden Entdeckung E. Beckmann's über die Einwirkung von FünffachChlorphosphor auf das Diphenylacetoxim lässt darüber kaum einen Zweifel übrig. Beckmann hat gezeigt, dass das Diphenylacetoxim mit Chlorphosphor nicht, wie man erwarten sollte, das Chlorid 
$\mathrm{C}_{6} \mathrm{H}_{5} \cdot \mathrm{C} \cdot \mathrm{C}_{6} \mathrm{H}_{5}$

$\dot{\mathrm{N}} . \mathrm{Cl}$ erzeugt, sondern dass es - durch eine der überraschendsten intramolecularen Umlagerungen - in das, diesem isomere Benzanilidchlorid $\mathrm{C}_{6} \mathrm{H}_{5} \cdot \mathrm{C} \cdot \mathrm{N} \cdot \mathrm{C}_{6} \mathrm{H}_{5}$ $\dot{\mathrm{Cl}}$

übergeht; dieses lieferte, mit Wasser zersetzt, dann Benzanilid.

In derselben Weise liefert unser Oxim durch Sprengung der Kohlenstoff bindungen und Ersatz derselben durch Stickstoff-Kohlenstoffrerkettung das

Isopropylamid der Isobuttersäure,

d. h. die Verbindung ${ }_{\text {sich in das isomere }}^{\mathrm{CH}_{3}}{ }_{\mathrm{CH}_{3}}^{\mathrm{CH}_{3}} \mathrm{CH} . \mathrm{CO} \cdot \mathrm{NH} \cdot \mathrm{CH}_{\mathrm{CH}_{3}}{ }_{\mathrm{CH}_{3}}^{\mathrm{CH}_{3}}$ und nichts

anderes als dies ist der von uns beschriebene flüchtige und krystallisirbare Körper. Dass sich derselbe aus dem als $Z$ wischenproduct entstandenen Aether $\mathrm{CH}_{3}$<smiles>CCC(C)OC#CC#CC(C)C</smiles>
bildet,

steht fest. Welcher Art aber der Verlauf der Atomrerschiebung ist, das bleibt bier so dunkel, wie bei der Beckmann'schen Entstehungsweise des Benzanilidchlorids.

Ist die oben entwickelte Ansicht die richtige, so musste sich der neue Körper leicht durch Synthese erbalten lassen, und um dies zu coustatiren, haben wir die

Einwirkung von Isobutyrylchlorid auf Isopropylamin studirt. Die erste Aufgabe, welche sich bierbei stellte, war die

Gewinnung von Isopropylamin.

Diese bisher so schwer zugängliche Base kann ohne Schwierigkeit unter Benutzung der Beobachtungen von Emil Fischer, Tafel und namentlich von Heinrich Goldschmidt gewonnen werden. Der letztere hat ganz kürzlich gezeigt, dass die Oxime mit 21/2 proc. Natriumamalgam in alkobolisch-essigsaurer Lösung reducirt, Amine geben.

Nun ist kaum irgend ein Oxim so leicht darstellbar, als das schön krystallisirende Dimethylacetoxim, $\mathrm{CH}_{3} \cdot \mathrm{C}(\mathrm{NOH}) . \mathrm{CH}_{3}$. Durch Reduction desselben, nach der Methode H. Goldschmidt's, erhielten wir das für die Versuche erforderliche Isopropylamin in genügender Menge, um die Synthese zu ermöglichen. Eine ätherische Lösung ron Isopropylamin rerwandelt sich mit Isobutyrylchlorid ganz glatt in 
eine Verbindung, welche in Ansehen, Löslichkeit, Krystallisation, Schmelz- und Siedepunkt sowie chemischen Eigenschaften mit unserer Base durchaus identisch ist.

Die Frage nach der Natur dieser Base ist somit in entscheidender Weise beantwortet. Was aber noch der Aufklärung bedarf, ist der folgende Punkt: Wie mitgetheilt, entsteht ausser der farblosen, krystallisirenden und völlig geruchloseu Verbindung stets ein $\mathrm{Oel}$ von intensivstem Alkaloügeruche. Ueber die Natur dieser Base Hypothesen anzustellen ist leicht, dieselben $z u$ beweisen aber schwierig, $\mathrm{da}$, wie gesagt, die Base nur in geringer Menge entsteht und von dem Rest an Krystallen, welche sie noch aufgelöst entbält, vorläufig nicht hat befreit werden können. Die Frage nach der Natur dieser basischen Substanz soll den Gegenstand weiterer Untersuchungen bilden, welche in hiesigem Laboratorium in Angriff genommen werden.

Schliesslicb sei bemerkt, dass es Eigenthünlichkeit der Oxime mit tertiären Wasserstoffatomen ist, Reactionen wie die hier beschriebene zu geben. Die Oxime:

$$
\left.\underset{\text { (Isopropylmethylacetoxim) }}{\underset{\mathrm{CH}_{3}}{\mathrm{CH}_{3}}>\mathrm{CH} . \mathrm{C}(\mathrm{NOH}) . \mathrm{CH}_{3}} \text { und } \underset{\text { (Isopropylphenylacetoxim) }}{\mathrm{CH}_{3}} \mathrm{CH}_{3}>\mathrm{CH} . \mathrm{C}(\mathrm{NOH}) . \mathrm{C}_{6} \mathrm{H}_{5}{ }^{\text {) }}\right)
$$

verhalten sich ganz analog; sie geben Substanzen von basischem Charakter, anstatt der zu erwartenden Acetyläther oder neben diesen Dagegen werden die Oxine des Benzophenons und Acetophenons

$$
\mathrm{C}_{6} \mathrm{H}_{5} . \mathrm{C}(\mathrm{NOH}), \mathrm{C}_{6} \mathrm{H}_{5} \text { and } \mathrm{C}_{6} \mathrm{H}_{5}, \mathrm{C}(\mathrm{NOH}) . \mathrm{CH}_{3}
$$

durch Chloracetyl einfach in Acetyläther verwandelt. Der erstere ist schon vor Jahren von E. Spiegler im Laboratorium des einen von uns in Zürich dargestellt worden. Die letztere Verbindung bat Hr. Rattner im hiesigen Laboratorium dargestellt; sie bildet Krystalle,

1) Das Isopropylphenylacetoxim, dargestellt von Hrn. Rattner, bildet (aus Ligroîn umkrystallisirt) Blättchen, welche bei $58^{\circ}$ schmolzen. Bei der Analyse lieferten

$0.1113 \mathrm{~g}$ Substanz $8.3 \mathrm{ccm}$ feuchten Stickstoff bei $16^{\circ}$ und $764 \mathrm{~mm}$ Druck. Berechnet

$$
\begin{aligned}
& \text { fir } \underset{\mathrm{CH}_{3}}{\mathrm{CH}_{3}}>\mathrm{CH} . \mathrm{C}(\mathrm{NOH}) . \mathrm{C}_{6} \mathrm{H}_{5} \quad \text { Gefunden } \\
& \begin{array}{lll}
\mathrm{N} & 8.64 & 8.74 \mathrm{pCt} .
\end{array}
\end{aligned}
$$

Acetylchlorid wirkt auf Isopropylphenylacetoxim sehr heftig. Wird wie oben beim Diisopropylacetoxim verfahren, so erbält man, ausser viel Harz, wenige Krystalle, die bei $115^{\circ}$ schmelzen und einen basischen Charakter zeigen.

Wurde während der Reaction das Acetylchlorid abgekühlt, so entstand ein Gemisch aus dem Acetylăther und unverāndertem Oxim. 
welche (aus Ligroïn umkrystallisirt) bei $53^{\circ}$ schmelzen und ergab bei der Analyse:

$0.141 \mathrm{~g}$ Substanz lieferten $10.2 \mathrm{ccm}$ feuchten Stickstoff bei $15^{\circ} \mathrm{C}$. und $745 \mathrm{~mm}$ Druck.

$$
\begin{array}{cl}
\multicolumn{2}{c}{\begin{array}{c}
\text { Berechnet } \\
\text { für } \mathrm{C}_{6} \mathrm{H}_{5} . \mathrm{C}\left(\mathrm{N} .0 . \mathrm{C}_{2} \mathrm{H}_{3} \mathrm{O}\right) . \mathrm{CH}_{3}
\end{array}} \\
\mathrm{~N} \quad 7.91 & \quad \text { Gefunden } \\
& 8.29 \mathrm{pCt} .
\end{array}
$$

Göttingen, Universitäts-Laborutoriun.

107. F. Münchmeyer: Zur Kenntniss der Reactionen einiger Disldehyde und Ketone.

(Eingegangen am 18. Februar; mitgeth. in der Sitzung v. Hrn. F. Tiemann.)

Nachdem ich in Heft 11 der vorjährigen Berichte gezeigt habe, dass sich ein Gesetz in Beziehung anf die Einwirkung von Hydroxylamin auf Biketone vor der Hand noch nicht aufstellen lässt, jedenfalls aber die früher aufgestellte Regel, dass nur diejenigen Diacetone, welche die Carbonylgruppen mit einander verbunden enthalten, mit 2 Molekülen Hydroxylamin reagiren, als gänzlich beseitigt anzusehen ist, war es interessant, das Verhalten von Dialdebyden gegen Hydroxylamin zu studiren. - Hierzu wurde ich noch besonders durch folgenden Umstand veranlasst: Westenberger hat gezeigt, dass sich Terephtalaldehyd mit 2 Molekülen Hydroxylamin zu Terepbtalaldoxim rerbindet, und dass dies Aldoxim, abweichend von allen seinen Analogen, mit Chloracetyl nicht Terephtalnitril sondern einen sehr beständigen Acetylester liefert. Diese merkwürdige Beobachtung, von deren völliger Richtigkeit erst kürzlich wieder Hr. Zelinsky im hiesigen Laboratorium sich überzeugt hat, macht es erwünscht, das Verhalten eines isomeren Dialdoxims gegen Chloracetyl kennen zu lernen.

Einwirkung von Hydroxylamin auf Isophtalaldebyd.

Der Isophtalaldehyd ist von Hrn. Dr. Fa u st in Göttingen zuerst rein dargestellt worden. Die ältere Angabe, die den Körper als Oel hinstellt, muss sich auf ein ganz unreines Präparat beziehen; das mir von Hrn. Dr. Faust freundlichst überlassene bestand aus Krystallblättchen, die genau wie Benzoësăure aussehen, und welche alle Farbenreactionen der Aldehyde in der ausgezeichnetsten Weise zeigten und mit Kaliumpermanganat reichlich reine Isophtalsäure lieferten. Schmelz- 